\title{
Characterization and Evaluation of Multiple Biological Activities of Silver Nanoparticles Fabricated from Dragon Tongue Bean Outer Peel Extract
}

This article was published in the following Dove Press journal:

International Journal of Nanomedicine

Jayanta Kumar Patra (iD)

Han-Seung Shin $\mathbb{D}^{2}$

Gitishree Das $\mathbb{D}^{1}$

'Research Institute of Biotechnology \& Medical Converged Science, Dongguk University-Seoul, Gyeonggi-do, I0326, Republic of Korea; ${ }^{2}$ Department of Food Science and Biotechnology, Dongguk University-Seoul, Gyeonggi-do, 10326, Republic of Korea
Correspondence: Gitishree Das Research Institute of Biotechnology \& Medical Converged Science, Dongguk University-Seoul, Gyeonggi-do, 10326 Republic of Korea

Email gitishreedas@gmail.com
Background: The dragon tongue beans are a legume belonging to the Fabaceae family, are rich in protein, starch, fiber, and other micronutrients that have numerous health-promoting benefits. Its peel commonly the waste parts also contains lots of bioactive compounds.

Materials and Methods: In the current research, dragon tongue bean peels (DtbP) extract is tested for the existence of phytochemicals. Ag nanoparticles are biosynthesized using DtbP extract. The generated DtbP silver nanoparticle characterization was accomplished using UVVis spectral analysis, FTIR spectral analysis, SEM analysis, EDX analysis, XRD analysis, zeta potential, and DLS study. Furthermore, comparative assessment on multi-biological activities of the biosynthesized $\mathrm{Ag}$ nanoparticles was accomplished by employing cytotoxicity (inhibition against $\mathrm{HepG}_{2}$ cancer cells), antidiabetic ( $\alpha$-glucosidase inhibition assay), and antioxidant (free-radical scavenging) analysis.

Results: The characterization result of the DtbP-AgNPs demonstrated that the AgNPs synthesized within $24 \mathrm{~h}$. The AgNPs are nearly spherical. The biological effect assay of AgNPs displayed that DtbP-AgNPs is having significant cytotoxicity, antidiabetic, and moderate antioxidant effect. This study results as a whole report the biosynthesis of DtbPAgNPs utilizing the legume dragon tongue bean waste peel and assessment of their multiple biological activities. The synthesized DtbP-AgNPs could serve as a potential candidate in the pharmaceutical industries in the formulation of drugs for the treatment of several medical ailments concerning cancer, diabetes, etc.

Keywords: antidiabetic, antioxidant, cytotoxicity, legume peel, silver nanoparticles, green nano-synthesis

\section{Introduction}

Nano-scaled technology offering distinctive features like their large surface area to surface volume ratio, small size, chemical, mechanical, magnetic, and optical properties. Nanotechnology prompts the use of novel metallic nanoparticles intended for the natural treatment of cancerous cells, bio-imaging, identification of diseases, and particular drug delivery. It has extensive applications in numerous sectors like food, biomedicine, agriculture, and cosmetic industries that have arisen as one of the huge arenas of research. ${ }^{1-3}$

At present, the synthesis of plant-mediated metal (Ag, $\mathrm{Au}, \mathrm{Zn}$ ) nanoparticles is the main point of attraction among current researchers due to its unique 
characteristics higher optoelectronic property, and biocompatibility. $^{2-4}$ Biosynthesis methods are recently used to synthesize nanoparticles by utilizing natural (reducing, capping, and stabilizing) agents. ${ }^{4-6}$ Using plant material synthesis of metal NPs like AgNPs is a non-toxic, harmless, economical, and eco-friendly method of nanoparticle synthesis. ${ }^{3,7}$ Silver is the foremost commercialized nano-particle with around five hundred tons of AgNPs produced nanoparticles/year and anticipated to increase shortly (Neha, 2019).

In the fabrication of NPs, there has been very rare usage of daily food waste resources from the domestic or food manufacturing industries. During the processing of plant food materials, the produced huge amount of byproducts throughout the processing of vegetables and fruits aggregates to an ecological problem owing to their high volume. The preventive methods to decrease the waste amount, the highly appropriate alternative is to reuse the waste resources into other materials or energy which can be utilized in numerous applications in pharmaceutical, cosmetics, and food trades. ${ }^{8}$ In the present day, it would be advantageous if food waste resources may well recycled and utilized as reducing agents for generating nanomaterial. To date to produce nanomaterials, several attempts have been made by a variety of biological waste resources to generate different types of nanomaterials that could be of low cost, eco-friendly, and extremely active for usage in the manufacture of pharmaceuticals and biomedicines. ${ }^{8}$

Previously, various studies have also reported vegetable waste peel aqueous extract mediated silver nanoparticles including our previous findings like Pisum sativum, ${ }^{8}$ and others like Momordica charantia, ${ }^{9}$ and Raphanus sativus $^{10}$ lime, ${ }^{11}$ etc. The eco-friendly and economical vegetable and fruit peels are generally considered waste products. However, research investigations on vegetable and fruit peels report the existence of vital phytochemicals and revealed their various biological activities like antioxidant, anti-inflammatory, and antimicrobial potential, etc., which can be utilized for therapeutic applications, in the health, food, and textile industries. ${ }^{12-16}$ Legumes including beans and peas are sources of plant proteins, vitamins, phenolic acids, flavonoids, amino acids, carbohydrates with diverse nutritive characteristics and are used globally in traditional medicines. ${ }^{17,18}$ The protein of beans can promote the reduction of cholesterol and the regulation of diabetes. The phenolic of beans can promote several benefits like reducing the incidence of cardiovascular and cancer diseases. It has also anti-inflammatory, antioxidant, antidiabetic, anticancer, antifungal, and antibacterial properties. ${ }^{17,19}$ Dragon tongue beans (Dtb) are botanically a cultivar of Phaseolus vulgaris species and a member of the family Fabaceae. The Dtb are the source of vitamins (A, C, K), proteins, carbohydrates, fibers, potassium, iron, calcium, and manganese, etc. The beans are the key food among the Fabaceae family with various bioactive compounds. $^{20,21}$ The beans have a great biological and antioxidant perspective which can be advantageous to the health of humans. ${ }^{21}$

There is a very rare report regarding the dragon tongue bean waste peel extract mediated fabrication of AgNPs and its biological properties. This study is economical and eco-friendly, hence the current research considered the use of the legume Dtb waste peels for the synthesis of AgNPs and assessment of their multiple potential such as cytotoxicity, anti-diabetic, and antioxidant effects.

\section{Materials and Methods}

\section{Materials, Preparation, and the DtbP Extract Phytochemical (Primary) Screening}

The dragon tongue bean was obtained from the Goyang-si marketplace (Republic of Korea). The primary phytochemical screening of DtbP extract was done for the existence of anthraquinones, flavonoids, proteins, amino acids, and carbohydrates using the standard protocols. Anthraquinones test: DtbP powdered sample $6 \mathrm{~g}$ was taken in a conical flask and benzene $(10 \mathrm{~mL})$ was added to it (soaked for $10 \mathrm{~min}$ ) and filtered. Ammonia solution $(10 \%), 10 \mathrm{~mL}$ was further mixed to it continuously for 30 seconds. In the ammonia phase change of color of the test solution to red, or pink-violet color specified the anthraquinones existence. ${ }^{22}$ Flavonoids test: In a test tube DtbP extract $(1 \mathrm{~mL})$ was taken and $1 \mathrm{~mL}$ of dilute $\mathrm{NaOH}$ was treated to it. The presence of a gloomy precipitate confirms the existence of flavonoids. ${ }^{23}$ Proteins and Amino acids: The proteins and amino acids in the DtbP extract were determined by the methods described by Sadasivam and Manickam, ${ }^{24}$ Ninhydrin solution (about $0.5 \mathrm{~mL}$ ) was added to $2 \mathrm{~mL}$ of the solvent extracts and boiled for 2 min and then cooled, the appearance of blue color confirms the presence of proteins and amino acids. Carbohydrate test: In a test-tube $2 \mathrm{~mL}$ of DtbP extract was taken, 2 drops of 5\% 1-naphthol in alcohol (Molisch's reagent) were added and mixed properly. 
Then the concentrated $\mathrm{H}_{2} \mathrm{SO}_{4}(1 \mathrm{~mL})$ was added to the side of the test tube. The appearance of a purple or redcum violet ring at the junction of the two liquid layers indicates the existence of carbohydrates. ${ }^{25}$

\section{DtbP Aqueous Extract Fabricated AgNPs Synthesis}

The dragon tongue bean peels (DtbP) were washed properly, drain out the water completely, and chopped into pieces. The chopped up waste peels $(100 \mathrm{~g})$ were taken in a 1 Lt Erlenmeyer flasks with $500 \mathrm{~mL} \mathrm{DDH}_{2} \mathrm{O}$, boiled with continuous stirring for $20 \mathrm{~min}$ and then cooled down, and filtered using a filter paper, and then put in storage at $4^{\circ} \mathrm{C}$ till use. ${ }^{8}$ For biosynthesis of AgNPs, briefly, $180 \mathrm{~mL} 1$ $\mathrm{mM} \mathrm{AgNO}_{3}$ solution was taken in a $500 \mathrm{~mL}$ of Erlenmeyer flasks and to it $20 \mathrm{~mL}$ of aqueous DtbP extract was added slowly dropwise with continuous stirring till complete $20 \mathrm{~mL}$ of the DtbP extract are mixed into the mixture. $^{26}$ The DtbP-AgNPs synthesis was witnessed by monitoring the gradual alteration in the pigment of the solution (test reaction solution) with respect to time period. The mixture solution was kept for $24 \mathrm{~h}$ and then it was centrifuged (at 10,000 rpm, 30 minutes). The supernatant was thrown away and the pallet was collected and cleaned with $\mathrm{DDH}_{2} \mathrm{O}$. The cleaning process was repeated 3-4 times, to remove the unreacted aqueous DtbP extract from the synthesized DtbP-AgNPs. In the concluding step, the generated pellets were dehydrated $\left(55^{\circ} \mathrm{C}\right)$ and well-preserved for further study.

\section{Synthesized DtbP-AgNPs Characterization}

The synthesized DtbP-AgNPs were characterized through UV-VIS spectrum analysis, FTIR, SEM, EDX, XRD, zeta potential, and DLS study through particular instruments using a standard protocol detailed in the previously published research reports. ${ }^{27}$ To generate DtbP silver NPs, the bio-reduction of the $\mathrm{Ag}+$ ions was evaluated by calculating the absorption spectra of the test reaction through a UVVIS spectrophotometer (Mutiskan Go; Thermo Scientific, Waltham, MA, USA) at $2 \mathrm{~nm}$ resolution in between 300-700 nm (for $24 \mathrm{~h}$ ). The color of the reaction solution was also documented by each time interval. Using an XRD machine (X'Pert MRD; PANalytical, Almelo, The Netherlands, set up with $\mathrm{Cu} \mathrm{K} \alpha$ radians at $40 \mathrm{~mA}$ and 30 $\mathrm{kV}$ at an angle of $2 \theta$ ) the nature of the bio-generated DtbPAgNPs was determined. On the glass slides (sample holder) the powdered AgNPs were loaded by evenly spreading following the standard procedure as stated ${ }^{27}$ and studied using the inbuilt software. The bio-generated DtbP-AgNPs were investigated for their surface morphology and elemental composition through the SEM and EDX analyzer. The DtbP silver NPs were spread over the glass slide using a carbon tape and sputter-coated with platinum before observation, through an ion coater apparatus for 2 min under SEM analyzer (Hitachi S-3000N, Tokyo, Japan). By using the technique stated by Zhou et $\mathrm{al}^{28}$ and Patra et $\mathrm{al}^{29}$ using an EDX detector allied with the SEM device, the elemental structure of the DtbP silver NPs was also detected. The FT-IR spectra of both AgNPs and the aqueous DtbP extract was evaluated by FT-IR spectrophotometer (Spectrum TwoTM FT-IR Spectrometer; PerkinElmer, MA, USA) at wavelengths ranged between $400-4000 \mathrm{~cm}^{-1}$. At the sample loading point, a pinch or $2 \mu \mathrm{L}$ of the DtbP extract was placed and analysed. The presence of various functional groups that participate in the NP synthesis was assessed by several modes of vibration. ${ }^{27}$ The bio-generated DtbP-AgNPs was characterized for their size distribution through DLS (Dynamic Light Scattering) and evaluated for their Zeta potential (Malvern Zetasizer Nano-ZS machine, Malvern, UK) to confirm the size and charge of DtbP-AgNPs by following the earlier published protocol of Clogston and Patri et $\mathrm{al}^{30}$ at $25^{\circ} \mathrm{C}$ temperature. Before analysis, the sample was sonicated and diluted using $\mathrm{DDH}_{2} \mathrm{O}$ in $1: 10$ concentration.

\section{Synthesized DtbP-AgNPs Antidiabetic, Antioxidant, and Cytotoxicity Effects}

The $\alpha$-glucosidase inhibition evaluation of the biosynthesized DtbP-AgNPs was accomplished by the standard procedure earlier reported by. ${ }^{28,31}$ The DtbP-AgNPs was liquefied in methanol and sonicated (using a water bath). $10 \mu \mathrm{g} / \mathrm{mL}$ DtbP-AgNPs sample for the test was moved to a 96-well plate and diluted successively with sodium phosphate buffer (0.02 M, pH 6.9). $\alpha$ - glucosidase at a concentration of $0.5 \mathrm{U} /$ $\mathrm{mL}$ was added to $50 \mu \mathrm{L}$ of the final volume. Next kept for incubation (10 $\mathrm{min})$ at room temperature. Further, P-nitrophenyl-glucopyranoside $(50 \mu \mathrm{L}, 3.0 \mathrm{mM})$ was taken as substrate. At $37^{\circ} \mathrm{C}$, the reaction mixture was kept for incubation for $20 \mathrm{~min}$. Later, the reaction was stopped by addition of $50 \mu \mathrm{L}$ aliquot of sodium carbonate $(0.1 \mathrm{M})$. Through a plate reader, the value (optical density) of the test solution (reaction mixture) was accessed at $405 \mathrm{~nm}$ 
(wavelength). The test plates contained positive standards (control) such as substrate, buffer, and enzyme. The $\alpha$ glucosidase inhibition percentage was evaluated as below.

$$
\text { Inhibition }(\%)=\frac{A_{\text {control }}-A_{\text {sample }}}{A_{\text {control }}} \times 100
$$

Here "A $\mathrm{A}_{\text {sample" }}$ is the tested sample absorbance value, and "A $\mathrm{A}_{\text {Contol }}$ " is the control sample absorbance value.

The DtbP-AgNPs antioxidant activity results were calculated by DPPH, and reducing power (free radical) assays employing the protocol (standard). ${ }^{8,28}$ In brief, the DPPH scavenging potential of the DtbP-AgNPs was evaluated in terms of percentage scavenging and effective concentration $\left(\mathrm{IC}_{50}\right)$. The absorbance was recorded by Multiskan Go; Thermo Scientific, Waltham, MA, USA spectrophotometer. BHQ (Tert Butyl-hydroquinone) was used as a standard reference and solvent methanol as the reference blank. The reducing power effect is estimated as the absorbance value of the test reaction solution at $700 \mathrm{~nm}$ using the same Multiskan spectrophotometer.

The cytotoxicity effect of DtbP-AgNPs was evaluated against the $\mathrm{HepG}_{2}$ cancerous cells (Korea Cell Line Bank, Seoul, and the Republic of Korea). The DtbP-AgNPs test sample was diluted at $1000 \mu \mathrm{g} / \mathrm{mL}$ concentrations using Dulbecco's phosphate-buffered saline, sterilized by filter (syringe filter, $0.22 \mu \mathrm{m}$, Millipore, Billerica, Ma, USA). An EZ-Cytox kit (DoGenBio Co., Ltd., Seoul, and the Republic of Korea) was used to evaluate the cytotoxicity effect. The cell viability (live cell \%) and morphology exposed to the synthesized DtbP-AgNPs were estimated through the trypan blue exclusion experiment. ${ }^{32}$ The optical density of DtbP-AgNPs (suspended in DMEM) was scanned in between the wavelength range of 300-700 nm, before the treatment of HepG2 cancer cells. After 24 $\mathrm{h}$ exposition, the supernatant was substituted with a 110 $\mu \mathrm{L}$ fresh medium (consist of $10 \mu \mathrm{L}$ of EZ-Cytox solution) and incubated for about $20 \mathrm{~min}$ until the melon red color transformed to yellowish-orange color. The DtbP-AgNPs was aliquoted in a new 96 well plate just after incubation. By using a spectrophotometer, the absorbance was taken at $450 \mathrm{~nm}$ wavelength (Spectra Max 384 Plus; Molecular Devices, Sunnyvale, CA, USA). Also, the cell viability of the HepG2 cells exposed to the produced DtbP-AgNPs was estimated as a result of trypan blue exclusion analysis. Likewise, after $24 \mathrm{~h}$ of exposure, the supernatant was discarded and the cells were washed with $100 \mu \mathrm{L}$ DPBS immediately. Next $20 \mu \mathrm{L}$ of the fresh complete DMEM and trypan blue mixture (1:1) was added to the well. Then the viability of the cell was observed through an inverted microscope (DMI6000B; Leica, Wetzlar, Germany).

\section{Statistical Analysis}

The outcome of the bioactivity is shown as mean \pm standard deviation. The one-way analysis of variance was accomplished by (Duncan's test at a 5\% level of significance) by SPSS software version 25.0 software.

\section{Results and Discussion Green Synthesis of DtbP-AgNPs}

The green technique comes under the bottom-up method which comprises oxidation or reduction course catalyzed by catalytic microbial enzymes or plant phytochemicals. The essential phytochemicals comprise are flavones, terpenoids, anthraquinones, carbohydrates, etc. ${ }^{33,34}$ As a natural source, the waste peel extracts are harmless and have a group of active metabolites that assists in the reduction process of $\mathrm{Ag}^{+}$ions. The dragon tongue beans or pinto beans hold a substantial amount of phytochemicals. ${ }^{19}$

Mostly the vegetables are peeled before intake. Various research reports, that vegetable peels are having antidiabetic, cardio-protective, and antioxidant potentials. ${ }^{35-37}$ Peels are the main portion of the vegetables and fruits. Generally, these peels are waste products and their disposal is challenging. ${ }^{37-39}$ Shell or pod of grain legumes provides protective wrap to the developing seeds. ${ }^{39,40}$ Globally common beans are the major grain legumes. In varied common bean cultivar's polyphenols are the major bioactive compounds. Flavonoids, proanthocyanidin, and phenolic acids are the foremost polyphenols found in maximum bean cultivars. ${ }^{21,41}$ Commonly polyphenols in beans are known to have anti-tumor, antidiabetic, antimicrobial, anti-inflammatory, antioxidant, and anti-mutagenic activity, etc. $^{21,41}$ In the pharmaceutical and food industries phytochemicals can be employed for the development of healthy food and medicines. ${ }^{14}$ In this research, the common bean or legume waste peel extract mediated DtbPAgNPs was synthesized (Figure 1A and B). The AgNPs synthesis was visually observed, and it remained progressive with gradual variation of color from colorless to the dark brown color in DtbP-AgNPs (Figure 1B). ${ }^{42}$

\section{Characterization of Synthesized DtbP-AgNPs}

Phaseolus vulgaris L. broadly known as common beans is one of the main grain legumes worldwide. In varied common 


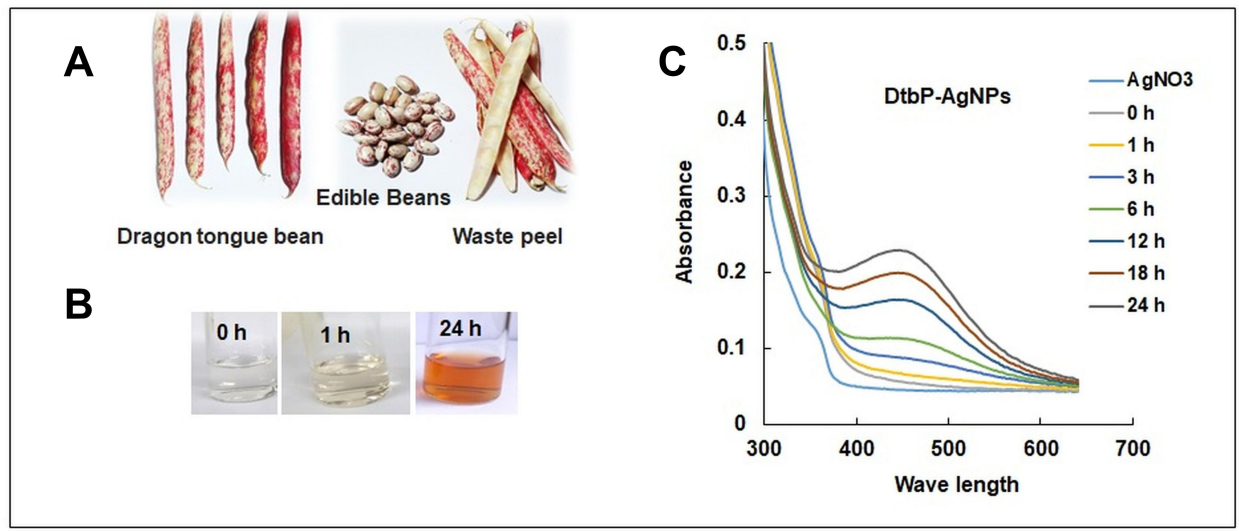

Figure I (A) Dragon tongue bean peel (DtbP); (B) Gradual change of DtbP extracts color in the progress of synthesis of DtbP-AgNPs (0-24 h); (C) UV-Vis spectral absorbance result of the DtbP-AgNPs.

bean cultivars, polyphenols are the predominant bioactive components with numerous bio-activities. The successfully synthesized DtbP-AgNPs were characterized through UVVis spectral study, SEM, EDX, XRD, FTIR, zeta potential, and DLS analysis. The biosynthesis of DtbP-AgNPs was established by the UV-Vis spectra analysis result. The DtbPAgNPs surface plasmon resonance value was detected at 450 $\mathrm{nm}$ wavelength (Figure 1C) which is analogous with earlier stated silver NPs synthesis results. ${ }^{8,43}$ The synthesized DtbPAgNPs were stable after $24 \mathrm{~h}$ as it shows the same absorption maxima further it was stored for 1 year and the absorption maxima were recorded, which showed no major changes. The result of the current research suggests that phytochemicals existing in the pod or shell of DtbP extract work as capping and reducing eco-friendly agents. The plant parts are natural, harmless, economical, eco-friendly, and possess various bioactive phytochemicals that help in the reduction process of $\mathrm{Ag}^{+}$ions. ${ }^{44}$

To determine the main functional groups existing in DtbP extract, which contribute to the NPs synthesis and stabilization course of the DtbP, the generated DtbPAgNPs were undergone FTIR analysis. Perhaps the exhibited FTIR study results, the peak values at 3310.09, 2118.56, 1075.98, $681.94 \mathrm{~cm}-1$ for DtbP-extract may shift to $\mathrm{cm}^{-1}$ in the case of DtbP-AgNPs 3321.40, 2135.53,1081.63 and 687.60 respectively (Figure 2A and $\mathrm{B})$. The variation in the absorption spectrum may suggest their contribution to DtbP-AgNPs synthesis. The DtbPAgNPs, FT-IR results revealed a deviation in absorption peaks employing varied or changed stretching modes amongst the extract and synthesized AgNPs (Figure 2A and B). The detected peaks in the case of DtbP-AgNPs at 3321.40 specifies $\mathrm{O}-\mathrm{H}$ stretch and $\mathrm{H}-\mathrm{a}$ bonded bond (belongs to alcohols and phenols functional groups). ${ }^{45}$ Similarly, the absorption peak value at $2135.53 \mathrm{~cm}-1$ (DtbP-AgNPs) indicates the attendance of the $\mathrm{C} \equiv \mathrm{N}$ stretch bond which comes under the nitriles functional group. Also, the absorption peak at $1081.63 \mathrm{~cm}^{-1}$ (DtbPAgNPs) designates the attendance of the $\mathrm{C}-\mathrm{O}$ stretch bond which comes under the functional groups (alcohols, esters, ethers, and carboxylic acids). Finally, the peak at 687.60 in DtbP-AgNPs specifies the existence of a strong $=\mathrm{C}-\mathrm{H}$ bend bond which comes under the alkenes functional group. ${ }^{45}$ The alteration in the absorption peak of the DtbP-AgNPs can be ascribed to the coating (capping) and equilibrium (stabilization) of the generated AgNPs. ${ }^{42}$

The morphology and the chemical pattern of the generated DtbP-AgNPs were detected by further SEM and EDX analysis. The DtbP-AgNPs were nearly spherical and in the nanometer range (Figure 3A and B). An analogous result was reported earlier. ${ }^{46}$ The elemental structure (configuration) of the generated AgNPs was identified through the EDX study. The consequence might well credit to the phytochemicals that exist in the used vegetable outer peel in the natural or green synthesis technique which may be supported in the coating (capping) and stabilization process of DtbPAgNPs. ${ }^{42}$ In the case of DtbP-AgNPs, the Ag was $26.55 \%$ (Figure 3B). The DtbP-AgNPs, XRD analysis displayed four distinct peaks in DtbP-AgNPs and confirmed the crystalline nature of the generated DtbP-AgNPs (Figure 4). The diffraction peaks were well noticed in the generated DtbP-AgNPs at 2 theta angles. The diffraction peaks for DtbP-AgNPs at $38.14,46.13,64.65$, and $76.98^{\circ}$ are equal to (111), (200), (220), and (311) correspondingly (Figure 4). These four observed peak values were correspondent to the fcc (facecentered cubic) phase of the Ag0 standard (JCPDS card No. 

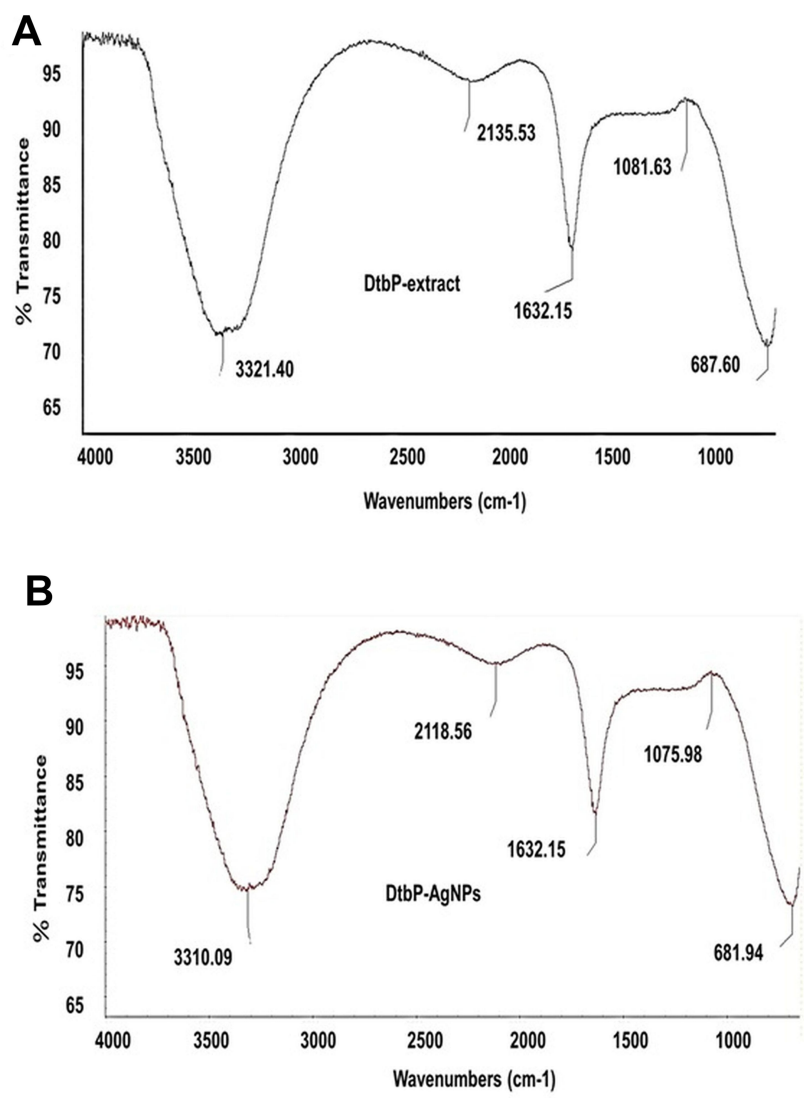

Figure 2 (A) FTIR data of DtbP-extract (B) FTIR data of DtbP-AgNPs.

04-0783). ${ }^{42,47}$ A parallel result was reported in earlier studies. $^{42}$ The zeta potential and DLS study of the biosynthesized DtbP-AgNPs was performed to know the surface charge and size distributions (hydrodynamic diameter) of the biosynthesized DtbP-AgNPs (Figure 5). The mean hydrodynamic diameter of DtbP-AgNPs exhibited as 78.02 (d.nm) (Figure 5A) and the zeta potential of DtbP-AgNPs displayed extremely negative $(-33.3 \mathrm{mV}$ ) (Figure $5 \mathrm{~B})$.

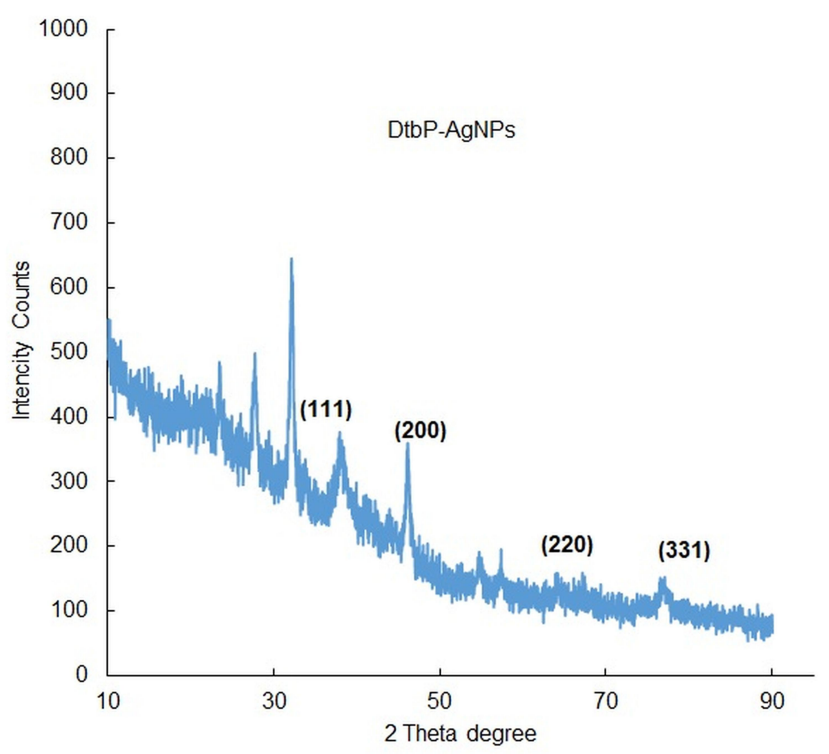

Figure $4 \times R D$ analysis of DtbP-AgNPs.

\section{Biological Activities of the Biosynthesized DtbP-AgNPs \\ Cytotoxicity (Anticancer) Activity of the Generated DtbP-AgNPs}

The NPs configuration makes them an outstanding method for tracking the wild cell growth initiated as a result of cancer. ${ }^{48}$ Nano drugs are exceptionally vigorous in the identification and management of cancer disease and concomitant syndromes. ${ }^{49}$ The cytotoxicity effect of DtbP-AgNPs was evaluated with different concentrations (1, 10, and $100 \mu \mathrm{g} /$ $\mathrm{mL}$ ) against $\mathrm{HepG}_{2}$ cancer cell lines and the results specified that the cell viability $\%$ of cancer cells $\left(\mathrm{HepG}_{2}\right)$ decreased with an increase of DtbP-AgNPs concentrations (Figure 6A and B). It was detected through an inverted microscope and was observed that the control was visualized with the maximum
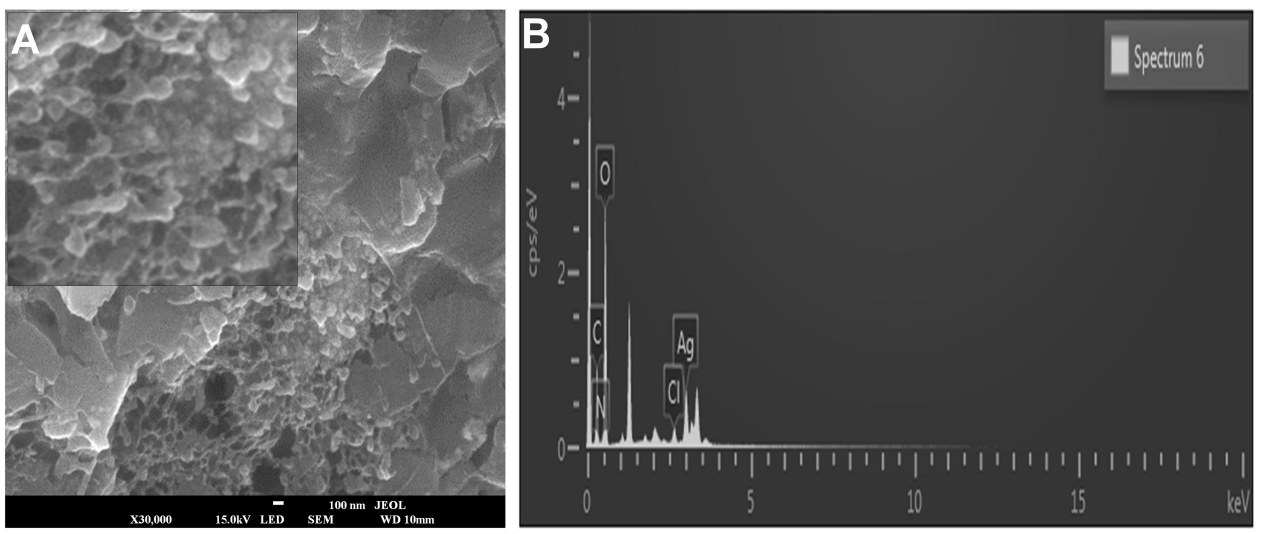

Figure 3 (A) SEM image of DtbP-AgNPs, (B) EDX image of DtbP-AgNPs. 


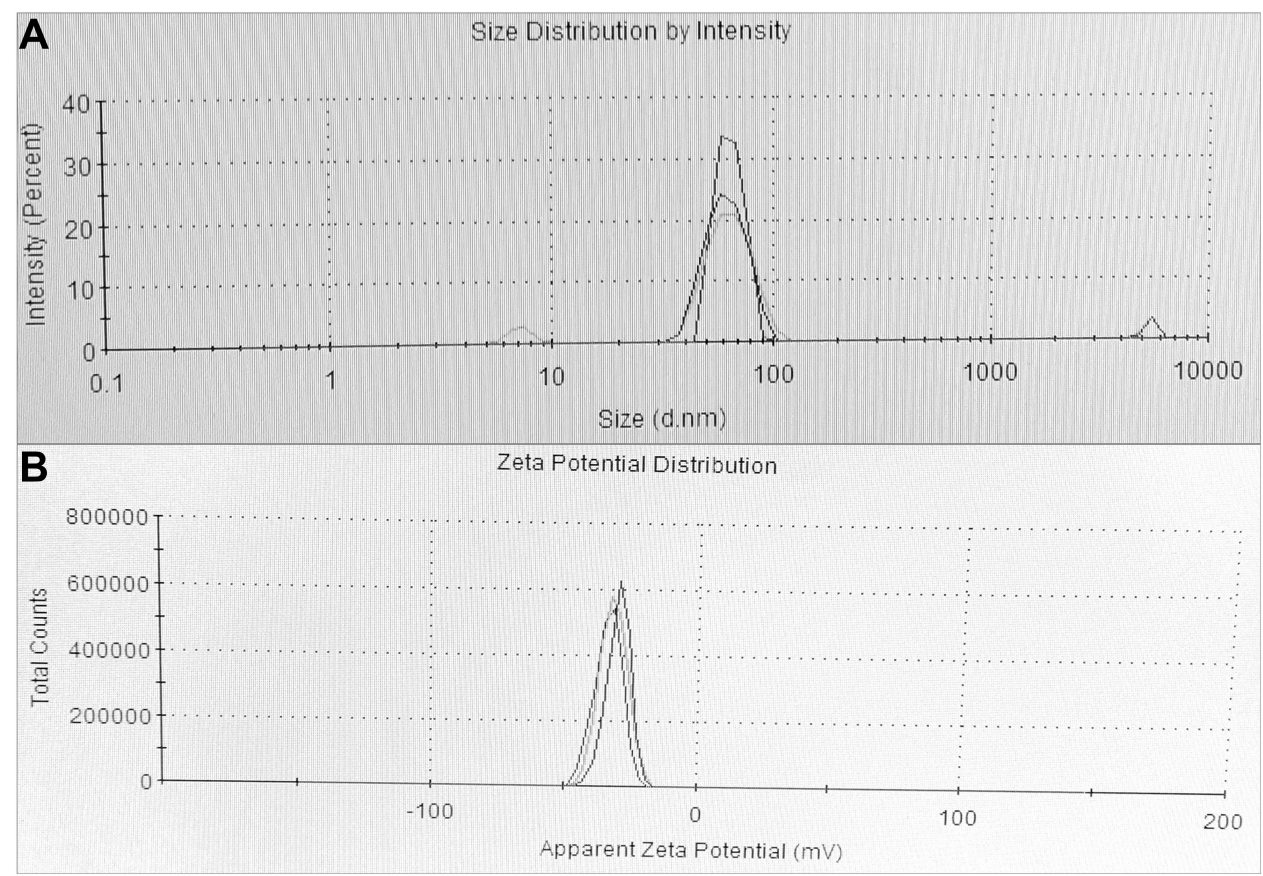

Figure 5 (A) Particle size distribution and (B) zeta potential study data of the DtbP-AgNPs.

number of live cells whereas in DtbP-AgNPs (treatment) the live cell percentage was gradually reduced with an increase in treatment (AgNPs) concentration. The generated DtbPAgNPs displayed significant cytotoxicity (anticancer) potential with a low $\mathrm{IC}_{50}$ value of $37.02 \mu \mathrm{g} / \mathrm{mL}$ (Table 1, Figure 6).

The probable cytotoxicity effect might be credited to the generated $\mathrm{Ag}$ nanoparticles which could have activated the generation of reactive oxygen species (ROS) in the cellular component resulting in the death of cancer cells. ${ }^{50}$ It has been assumed that the silver nanoparticles interact with the mitochondria and interferes with the function of transfer of cellular electron chain resulting in a rise in the level of ROS. ${ }^{51}$ Thus the oxidative stress produced by ROS might be considered as the foremost mechanism of toxicity against the cancer cell lines. It is anticipated that greater the cytotoxicity effect of the silver nanoparticle against cancer cell lines owing to the highest uptake of nanoparticles by the cell lines, as the cancer cell lines have an unusual metabolism and high percentage of proliferation, which in turn makes the cancer cell line more susceptible. ${ }^{52}$ In earlier research, analogous results are stated by. ${ }^{49,53}$ Besides, our previous research on the green pea peels extract mediated silver nanoparticle synthesis and its biological potential also displayed similar cytotoxicity potential. ${ }^{8}$

\section{Antidiabetic ( $\alpha$-Glucosidase Inhibition) Activity of Generated DtbP-AgNPs}

For the reduction of hyperglycemia, the carbohydrate digesting enzymes ( $\alpha$-glucosidase and $\alpha$-amylase) requires to be inhibited therapeutically, thus constraining the breakdown of carbohydrates into monosaccharides, which are the key donors to elevated blood sugar levels. ${ }^{54}$ Hence developing compounds with an inhibitory action towards carbohydratehydrolyzing enzymes may well beneficial to control diabetes. In this research, the DtbP-AgNPs displayed significant antidiabetic action dose-dependently. $\mathrm{The} \mathrm{IC}_{50}$ value of the DtbP-AgNPs was evaluated and presented in Table 1.

The DtbP-AgNPs exhibited promising antidiabetic potential (Figure 7) with a low $\mathrm{IC}_{50}$ value of $1.98 \mu \mathrm{g} / \mathrm{mL}$ (Table 1). The considerable antidiabetic effect of the DtbP-AgNPs (Figure 7) presented in this study is a sign of their efficiency in the control of diabetes. It is assumed that the AgNPs could have inhibited the specific enzymes (in this case $\alpha$ glucosidase) by binding to the other part of the enzyme, not to its active part and enzyme-substrate complex. ${ }^{55}$ Earlier it was also reported that the AgNPs could have reduced the enzyme levels, which are in charge of catalyzing the hydrolysis of complex carbohydrates, and improved the rate of usage of glucose which might be the reason for the antidiabetic effect of the generated AgNPs. ${ }^{56-58}$ In earlier research analogous results have been detailed for silver nanoparticles 


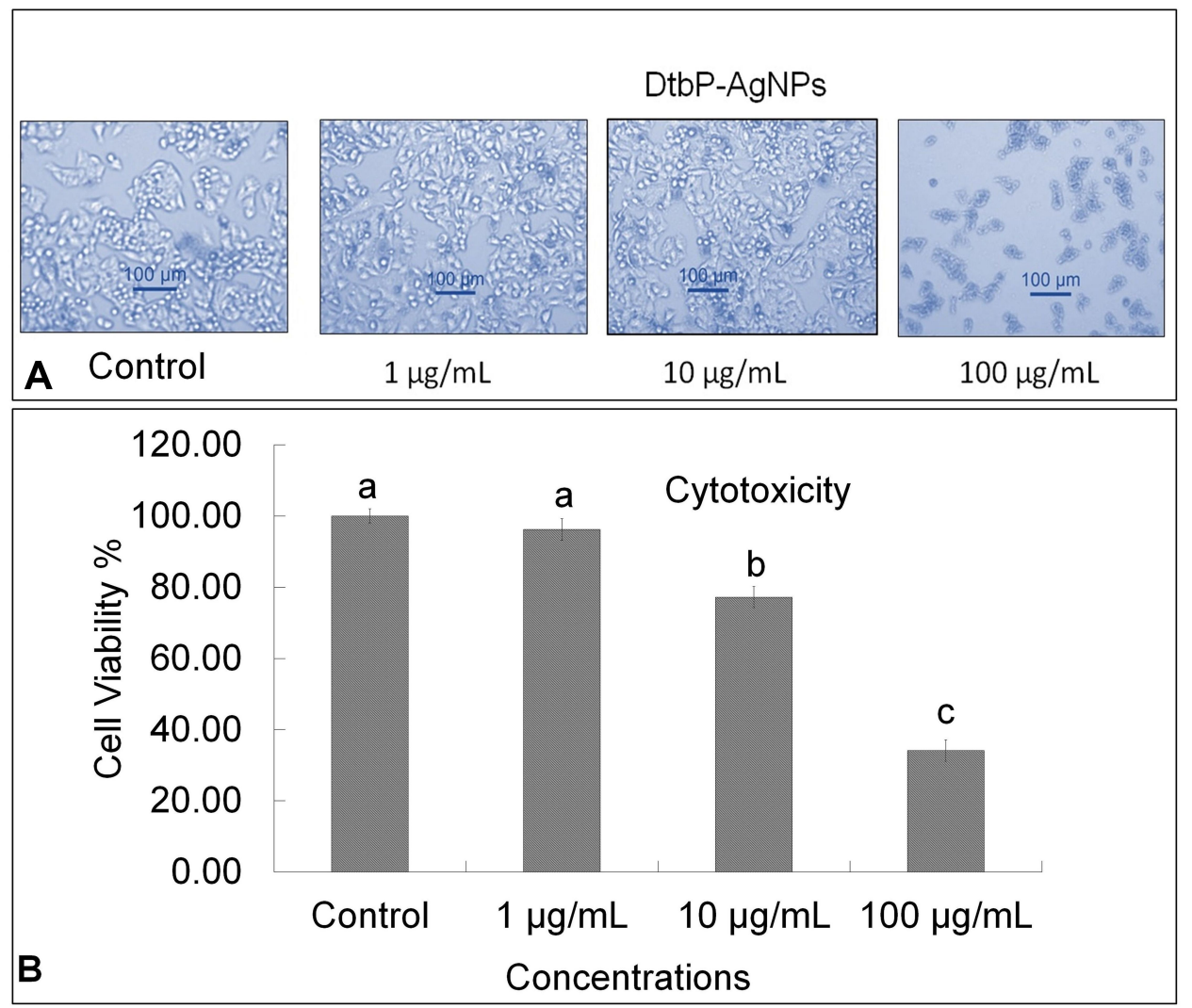

Figure 6 (A) Cytotoxicity effect: Result image of Control, DtbP-AgNPs (Dead cell \%), against HepG2 cancer cells. (B) Cytotoxicity (Cell viability \%) result of DtbP-AgNPs. Columns with different letters $(a, b, c)$ are statistically significant at $P<0.05$.

in terms of the $\alpha$-glucosidase activity. ${ }^{59}$ Further our previous research also displayed the significant antidiabetic activity of the green pea peel mediated Ag nanoparticles. ${ }^{8}$

\section{Antioxidant (Free Radical Scavenging) Action of Generated DtbP-AgNPs}

The free radical scavenging (antioxidant) effect of the DtbP-AgNPs was estimated through DPPH, reducing power (Figure 8), ABTS, and NOx antioxidant (Table 1) assays at $25-100 \mu \mathrm{g} / \mathrm{mL}$. The DtbP-AgNPs displayed

Table I IC 50 Values for Antioxidant, Antidiabetic, and Cytotoxic Assays of DtbP-AgNPs

\begin{tabular}{|l|l|l|}
\hline & Parameters & $\begin{array}{l}\text { IC }_{\mathbf{5 0}} \text { Value } \\
\text { DtbP-AgNPs } \\
(\boldsymbol{\mu g} / \mathbf{m L})\end{array}$ \\
\hline Antioxidant test & DPPH & 4.37 \\
& ABTS & 23.59 \\
& NOx & 23.05 \\
Antidiabetic test & Reducing (IC (-glucosidase value) & 88.37 \\
\hline Cytotoxicity test & & 1.98 \\
\hline
\end{tabular}

moderate antioxidant activity (Table 1). The reducing power $\mathrm{IC}_{50}$ value of DtbP-AgNPs was $88.37 \mu \mathrm{g} / \mathrm{mL}$ (Table 1). While for the ABTS scavenging assessment, the $\mathrm{IC}_{50}$ value of DtbP-AgNPs was $23.59 \mu \mathrm{g} / \mathrm{mL}$. For the NOx scavenging assay, the $\mathrm{IC}_{50}$ value of DtbP-AgNPs was

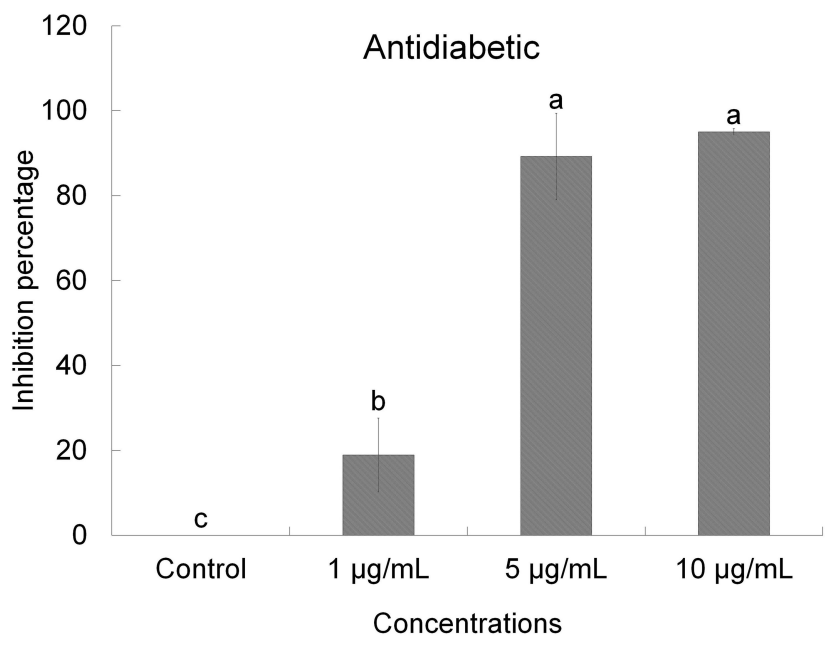

Figure 7 Antidiabetic ( $\alpha$-glucosidase) effect of DtbP-AgNPs. Columns with different letters $(\mathrm{a}, \mathrm{b}, \mathrm{c})$ are statistically significant at $P<0.05$. 

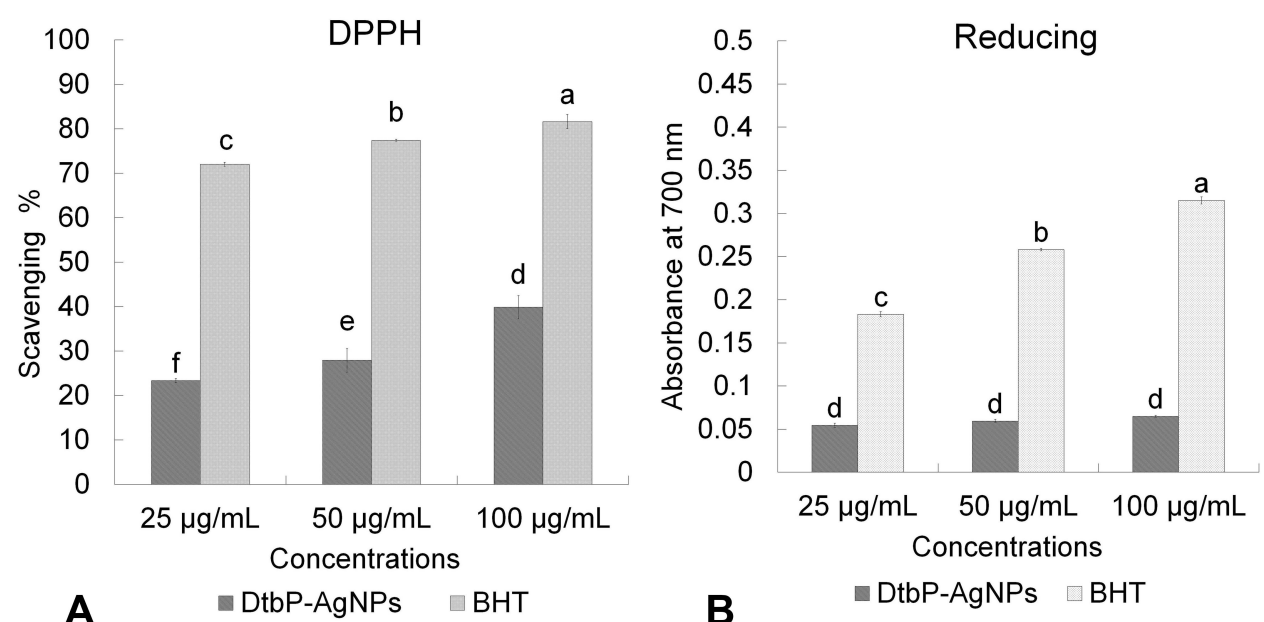

Figure 8 Antioxidant (A) (DPPH assay) and (B) (Reducing power assay) effect of DtbP-AgNPs. Columns with different letters (a,b,c,d,e,f) are statistically significant at $P<0.05$.

$23.05 \mu \mathrm{g} / \mathrm{mL}$. The $\mathrm{IC}_{50}$ value of DtbP-AgNPs was $4.37 \mu \mathrm{g} /$ $\mathrm{mL}$ for the DPPH scavenging (Figure 8) assay (Table 1). Numerous research has shown that the antioxidant activity of AgNPs is generated from plant extracts. ${ }^{60}$ It was also reported that the antioxidant effect of AgNPs is due to the capping ability of the biomolecules existing in bio-extracts to the NPs during the biosynthesis process. ${ }^{61}$ In this study, the resultant antioxidant activity may be due to the abundant active functional groups present in the DtbP extract which might have played an important role in the coating and stabilization of DtbP-AgNPs. ${ }^{62}$ Antioxidants are vital molecules required to neutralize the generation of excessive free radicals in an aerobic organism and subsequently protect the body against prompted oxidative stress-related ailments. ${ }^{55,63}$ The antioxidant potential of the synthesized AgNPs has been stated to have a relative advantage over the traditional antioxidant delivery system in terms of the targeted and controlled delivery, elevated bioavailability, and condensed protection of antioxidant agents. ${ }^{55,64}$

\section{Conclusions}

The legume Dtb outer waste peel extract was well utilized and was effective in the green synthesis of DtbP-AgNPs. The biological activities of the generated AgNPs were estimated. The DtbP-AgNPs demonstrated a noteworthy cytotoxicity and antidiabetic effects with a reasonable antioxidant activity, which possibly may be owing to the existence of the bioactive functional group of compounds in the DtbP extract. The study result proposes that the generated DtbP-AgNPs could be a favorable candidate in the pharmaceutical industries for the cure of potential health-related ailments.

\section{Acknowledgments}

JK Patra, H-S Shin, and G Das are gratefully acknowledging Dongguk University-Seoul, Republic of Korea, for the support. This work was supported by the National Research Foundation of Korea (NRF) grant funded by the Korea government (MSIT) (No. 2020R1G1A1004667), the Republic of Korea. The authors wish to thank whoever helped directly or indirectly in this research work.

\section{Author Contributions}

All authors contributed to data analysis, drafting or revising the article, have agreed on the journal to which the article will be submitted, gave final approval of the version to be published, and agree to be accountable for all aspects of the work.

\section{Funding}

This work was supported by the National Research Foundation of Korea (NRF) grant funded by the Korea government (MSIT) (No. 2020R1G1A1004667), the Republic of Korea.

\section{Disclosure}

The authors declare that there is no conflict of interest exists with this manuscript.

\section{References}

1. Zhang D, Liu T, Cheng J, et al. Controllable synthesis and characterization of tungsten disulfide nanosheets as promising nanomaterials for electronic devices. Ceramics Int. 2019;45(9):12443-12448. doi:10.10 16/j.ceramint.2019.03.177

2. Yaqoob SB, Adnan R, Khan RMR, Rashid M. Gold, silver, and palladium nanoparticles: a chemical tool for biomedical applications. Front Chem. 2020;8. doi:10.3389/fchem.2020.00376 
3. Hemlata PR, Singh AP, Tejavath KK, Tejavath KK. Biosynthesis of silver nanoparticles using cucumis prophetarum aqueous leaf extract and their antibacterial and antiproliferative activity against cancer cell lines. ACS Omega. 2020;5(10):5520-5528. doi:10.1021/acsomega. $0 \mathrm{c} 00155$

4. Neha N. Synthesis of silver nanoparticles and phytochemical analysis of Benincasa hispida peel extract. Int J Pharm Biol Sci. 2019;9 (3):329-343.

5. El-Nour KMA, Eftaiha A, Al-Warthan A, Ammar RA. Synthesis and applications of silver nanoparticles. Arab J Chem. 2010;3 (3):135-140. doi:10.1016/j.arabjc.2010.04.008

6. Alomar TS, Al-Masoud N, Awad MA, El-Tohamy MF, Soliman DA. An eco-friendly plant-mediated synthesis of silver nanoparticles: characterization, pharmaceutical and biomedical applications. Mater Chem Phys. 2020;249:123007. doi:10.1016/j.matchemphys.2020.123007

7. Aritonang HF, Koleangan H, Wuntu AD. Synthesis of silver nanoparticles using aqueous extract of medicinal plants' (Impatiens balsamina and Lantana camara) fresh leaves and analysis of antimicrobial activity. Int J Microbiol. 2019;2019:1-8. doi:10.1155/ 2019/8642303

8. Patra JK, Das G, Shin H-S. Facile green biosynthesis of silver nanoparticles using Pisum sativum L. outer peel aqueous extract and its antidiabetic, cytotoxicity, antioxidant, and antibacterial activity. Int $J$ Nanomedicine. 2019;14:6679. doi:10.2147/IJN. S212614

9. Shelar A, Sangshetti J, Chakraborti S, Singh AV, Patil R, Gosavi S. Helminthicidal and larvicidal potentials of biogenic silver nanoparticles synthesized from medicinal plant Momordica charantia. Med Chem (Los Angeles). 2019;15(7):781-789. doi:10.2174/157340641 5666190430142637

10. Fadel Q, Al-Mashhedy L. Biosynthesis of silver nanoparticles using peel extract of Raphanus sativus L. Biotechnol Ind J. 2017;13(1):120.

11. Irshad MS, Arshad N, Ahmed I, et al. Low-cost green recyclable biomaterial for energy-dependent electrical switching and intact biofilm with antibacterial properties. Sci Rep. 2020;10(1):1-16. doi:10.1038/s41598-020-71610-w

12. Santos C, Abreu C, Freire JM, Queiroz E, Mendonça MM. Chemical characterization of the flour of peel and seed from two papaya cultivars. Food Sci Technol. 2014;34(2):353-357. doi:10.1590/ fst.2014.0048

13. Khattak K, Rahman T. Analysis of vegetable's peels as a natural source of vitamins and minerals. Int Food Res J. 2017;24(1):292.

14. Sagar NA, Pareek S, Sharma S, Yahia EM, Lobo MG. Fruit and vegetable waste: bioactive compounds, their extraction, and possible utilization. Comprehen Rev Food Sci Food Saf. 2018;17(3):512-531.

15. Saleem M, Saeed MT. Potential application of waste fruit peels (orange, yellow lemon and banana) as wide range natural antimicrobial agent. J King Saud Univ Sci. 2020;32(1):805-810. doi:10.1016/j. jksus.2019.02.013

16. Kumar H, Bhardwaj K, Sharma R, et al. Fruit and vegetable peels: utilization of high value horticultural waste in novel industrial applications. Molecules. 2020;25(12):2812. doi:10.3390/molecules 25122812

17. Rungruangmaitree R, Jiraungkoorskul W. Pea, Pisum sativum, and its anticancer activity. Pharmacogn Rev. 2017;11(21):39. doi:10.4103/ phrev.phrev_57_16

18. Moghadam M, Salami M, Mohammadian M, Khodadadi M, EmamDjomeh Z. Development of antioxidant edible films based on mung bean protein enriched with pomegranate peel. Food Hydrocoll. 2020;104:105735. doi:10.1016/j.foodhyd.2020.105735

19. Carbas B, Machado N, Oppolzer D, et al. Nutrients, antinutrients, phenolic composition, and antioxidant activity of common bean cultivars and their potential for food applications. Antioxidants. 2020;9(2):186. doi:10.3390/antiox9020186
20. El-Batal AI, Gharib FAE-L, Ghazi SM, Hegazi AZ, Hafz AGMAE. Physiological responses of two varieties of common bean (Phaseolus vulgaris L.) to foliar application of silver nanoparticles. Nanomater Nanotechnol. 2016;6:13. doi:10.5772/62202

21. Alcázar-Valle M, Lugo-Cervantes E, Mojica L, et al. Bioactive compounds, antioxidant activity, and antinutritional content of legumes: a comparison between four phaseolus species. Molecules. 2020;25 (15):3528. doi:10.3390/molecules 25153528

22. Gul R, Jan SU, Faridullah S, Sherani S, Jahan N. Preliminary phytochemical screening, quantitative analysis of alkaloids, and antioxidant activity of crude plant extracts from Ephedra intermedia indigenous to Balochistan. Sci World J. 2017;2017:1-7. doi:10.11 $55 / 2017 / 5873648$

23. Sofowora A. Medicinal plants and medicine in Africa. John Whilley Spectr Books. 1993;120-123.

24. Sadasivam S, Manickam A. Biochemical Methods. 2nd ed. New Delhi: New age International; 2005.

25. Parekh J, Chanda S. In vitro antimicrobial activity and phytochemical analysis of some Indian medicinal plants. Turk J Biol. 2007;31 (1):53-58.

26. Patra JK, Baek K-H. Antibacterial activity and synergistic antibacterial potential of biosynthesized silver nanoparticles against foodborne pathogenic bacteria along with its anticandidal and antioxidant effects. Front Microbiol. 2017;8:167. doi:10.3389/fmicb.2017.00167

27. Iravani S, Korbekandi H, Mirmohammadi SV, Zolfaghari B. Synthesis of silver nanoparticles: chemical, physical and biological methods. Res Pharm Sci. 2014;9(6):385.

28. Zhou Y, Itoh H, Uemura T, Naka K, Chujo Y. Preparation of $\pi$ conjugated polymer-protected gold nanoparticles in stable colloidal form. Chem Commun. 2001;(7):613-614. doi:10.1039/b100636n

29. Patra JK, Das G, Kumar A, Ansari A, Kim H, Shin H-S. Photomediated biosynthesis of silver nanoparticles using the non-edible accrescent fruiting calyx of Physalis peruviana L. fruits and investigation of its radical scavenging potential and cytotoxicity activities. $J$ Photochem Photobiol B. 2018;188:116-125. doi:10.1016/j. jphotobiol.2018.08.004

30. Clogston JD, Patri A. Characterization of nanoparticles intended for drug delivery. Methods Mol Biol. 2011.

31. Butala MA, Kukkupuni SK, Venkatasubramanian P, Vishnuprasad CN. An ayurvedic anti-diabetic formulation made from Curcuma longa L. and Emblica officinalis L. Inhibits $\alpha$-amylase, $\alpha$-glucosidase, and starch digestion, in vitro. Starch-Stärke. 2018;70(5-6):1700182. doi:10.1002/star.201700182

32. Faedmaleki F, Shirazi FH, Salarian -A-A, Ashtiani HA, Rastegar H. Toxicity effect of silver nanoparticles on mice liver primary cell culture and HepG2 cell line. Iran J Pharm Res. 2014;13(1):235.

33. Prabhu S, Poulose EK. Silver nanoparticles: mechanism of antimicrobial action, synthesis, medical applications, and toxicity effects. Int Nano Lett. 2012;2(1):32. doi:10.1186/2228-5326-2-32

34. Kota S, Dumpala P, Anantha RK, Verma MK, Kandepu S. Evaluation of therapeutic potential of the silver/silver chloride nanoparticles synthesized with the aqueous leaf extract of Rumex acetosa. Sci Rep. 2017;7(1):1-11.

35. Shukla S, Anusha A, Archana K, et al. Antihypergluco-lipidemic and antioxidant activities in aqueous methanol extract of some vegetables peel: an in vitro analysis. PTB Rep. 2016;2(1).

36. Ramya S, Narayanan V, Ponnerulan B, Saminathan E, Veeranan U. Potential of peel extracts of Punica granatum and Citrus aurantifolia on alloxan-induced diabetic rats. Beni-Suef Univ J Basic Appl Sci. 2020;9(1):1-11. doi:10.1186/s43088-020-00049-9

37. Ben-Othman S, Jõudu I, Bhat R. Bioactives from agri-food wastes: present insights and future challenges. Molecules. 2020;25(3):510. doi:10.3390/molecules 25030510 
38. Jecu L. Solid state fermentation of agricultural wastes for endoglucanase production. Ind Crops Prod. 2000;11(1):1-5. doi:10.1016/ S0926-6690(99)00022-9

39. Verma N, Bansal MC, Kumar V. Pea peel waste: a lignocellulosic waste and its utility in cellulase production by Trichoderma reesei under solid state cultivation. Bioresources. 2011;6(2):1505-1519. doi:10.15376/biores.6.2.1505-1519

40. Tosh SM, Yada S. Dietary fibres in pulse seeds and fractions: characterization, functional attributes, and applications. Food Res Int. 2010;43(2):450-460. doi:10.1016/j.foodres.2009.09.005

41. Yang QQ, Gan RY, Ge YY, Zhang D, Corke H. Polyphenols in common beans (Phaseolus vulgaris L.): chemistry, analysis, and factors affecting composition. Comprehen Rev Food Sci Food Saf. 2018;17(6):1518-1539. doi:10.1111/1541-4337.12391

42. He Y, Wei F, Ma Z, et al. Green synthesis of silver nanoparticles using seed extract of Alpinia katsumadai, and their antioxidant, cytotoxicity, and antibacterial activities. RSC Adv. 2017;7 (63):39842-39851. doi:10.1039/C7RA05286C

43. Mahanty A, Mishra S, Bosu R, Maurya U, Netam SP, Sarkar B. Phytoextracts-synthesized silver nanoparticles inhibit bacterial fish pathogen Aeromonas hydrophila. Indian J Microbiol. 2013;53 (4):438-446. doi:10.1007/s12088-013-0409-9

44. Kota S, Dumpala P, Anantha RK, Verma MK, Kandepu S. Evaluation of therapeutic potential of the silver/silver chloride nanoparticles synthesized with the aqueous leaf extract of Rumex acetosa. Sci Rep. 2017;7(1):11566. doi:10.1038/s41598-017-11853-2

45. Kalaiyarasu T, Karthi N, Sharmila GV, Manju V. In vitro assessment of antioxidant and antibacterial activity of green synthesized silver nanoparticles from Digitaria radicosa leaves. Asian J Pharm Clin Res. 2016;9(1).

46. Ismail M, Gul S, Khan M, Khan MA, Asiri AM, Khan SB. Medicago polymorpha-mediated antibacterial silver nanoparticles in the reduction of methyl orange. Green Process Synth. 2019;8(1):118-127. doi:10.1515/gps-2018-0030

47. Jagtap U, Bapat V. Biosynthesis, characterization and antibacterial activity of silver nanoparticles by aqueous Annona squamosa L. leaf extract at room temperature. J Plant Biochem Biotechnol. 2013;22 (4):434-440. doi:10.1007/s13562-012-0172-8

48. Pugazhendhi A, Edison TNJI, Karuppusamy I, Kathirvel B. Inorganic nanoparticles: a potential cancer therapy for human welfare. Int J Pharm. 2018;539(1-2):104-111. doi:10.1016/j.ijpharm.2018.01.034

49. Oves M, Aslam M, Rauf MA, et al. Antimicrobial and anticancer activities of silver nanoparticles synthesized from the root hair extract of Phoenix dactylifera. Mater Sci Eng C. 2018;89:429-443. doi:10.1016/j.msec.2018.03.035

50. Venugopal K, Ahmad H, Manikandan E, et al. The impact of anticancer activity upon Beta vulgaris extract mediated biosynthesized silver nanoparticles (ag-NPs) against human breast (MCF-7), lung (A549) and pharynx (Hep-2) cancer cell lines. J Photochem Photobiol B. 2017;173:99-107. doi:10.1016/j.jphotobiol.2017.05.031

51. Singh RP, Ramarao P. Cellular uptake, intracellular trafficking and cytotoxicity of silver nanoparticles. Toxicol Lett. 2012;213 (2):249-259. doi:10.1016/j.toxlet.2012.07.009
52. Alkhulaifi MM, Alshehri JH, Alwehaibi MA, et al. Green synthesis of silver nanoparticles using Citrus limon peels and evaluation of their antibacterial and cytotoxic properties. Saudi J Biol Sci. 2020;27:3434-3441. doi:10.1016/j.sjbs.2020.09.031

53. Bhatnagar S, Kobori T, Ganesh D, Ogawa K, Aoyagi H. Biosynthesis of silver nanoparticles mediated by extracellular pigment from Talaromyces purpurogenus and their biomedical applications. Nanomaterials. 2019;9(7):1042. doi:10.3390/nano9071042

54. Nickavar B, Abolhasani L. Bioactivity-guided separation of an $\alpha$ amylase inhibitor flavonoid from Salvia virgata. Iran J Pharm Res. 2013;12(1):57.

55. Badmus J, Oyemomi S, Adedosu O, et al. Photo-assisted bio-fabrication of silver nanoparticles using Annona muricata leaf extract: exploring the antioxidant, anti-diabetic, antimicrobial, and cytotoxic activities. Heliyon. 2020;6(11):e05413. doi:10.1016/j.heliyon.2020.e05413

56. Sengottaiyan A, Aravinthan A, Sudhakar C, et al. Synthesis and characterization of Solanum nigrum-mediated silver nanoparticles and its protective effect on alloxan-induced diabetic rats. J Nanostruct Chem. 2016;6(1):41-48. doi:10.1007/s40097-015-0178-6

57. Balan K, Qing W, Wang Y, et al. Antidiabetic activity of silver nanoparticles from green synthesis using Lonicera japonica leaf extract. RSC Adv. 2016;6(46):40162-40168. doi:10.1039/C5RA24391B

58. Yarrappagaari S, Gutha R, Narayanaswamy L, et al. Eco-friendly synthesis of silver nanoparticles from the whole plant of Cleome viscosa and evaluation of their characterization, antibacterial, antioxidant and antidiabetic properties. Saudi $J$ Biol Sci. 2020;27:3601-3614. doi:10.1016/j.sjbs.2020.07.034

59. Govindappa M, Hemashekhar B, Arthikala M-K, Rai VR, Ramachandra Y. Characterization, antibacterial, antioxidant, antidiabetic, anti-inflammatory and antityrosinase activity of green synthesized silver nanoparticles using Calophyllum tomentosum leaves extract. Results Phys. 2018;9:400-408. doi:10.1016/j.rinp.2018. 02.049

60. Yousaf H, Mehmood A, Ahmad KS, Raffi M. Green synthesis of silver nanoparticles and their applications as an alternative antibacterial and antioxidant agents. Mater Sci Eng C. 2020;112:110901. doi:10.1016/j.msec.2020.110901

61. Hussain A, Mehmood A, Murtaza G, et al. Environmentally benevolent synthesis and characterization of silver nanoparticles using Olea ferruginea Royle for antibacterial and antioxidant activities. Green Process Synth. 2020;9(1):451-461. doi:10.1515/gps-2020-0047

62. El-Seedi HR, El-Shabasy RM, Khalifa SA, et al. Metal nanoparticles fabricated by green chemistry using natural extracts: biosynthesis, mechanisms, and applications. RSC Adv. 2019;9(42):24539-24559. doi:10.1039/C9RA02225B

63. Tan BL, Norhaizan ME, Liew W-P-P, Sulaiman Rahman H. Antioxidant and oxidative stress: a mutual interplay in age-related diseases. Front Pharmacol. 2018;9:1162.

64. Khalil I, Yehye WA, Etxeberria AE, et al. Nanoantioxidants: recent trends in antioxidant delivery applications. Antioxidants. 2020;9 (1):24. doi:10.3390/antiox9010024
International Journal of Nanomedicine

\section{Publish your work in this journal}

The International Journal of Nanomedicine is an international, peerreviewed journal focusing on the application of nanotechnology in diagnostics, therapeutics, and drug delivery systems throughout the biomedical field. This journal is indexed on PubMed Central, MedLine, CAS, SciSearch ${ }^{\mathbb{R}}$, Current Contents ${ }^{\mathbb{B}} /$ Clinical Medicine,
Journal Citation Reports/Science Edition, EMBase, Scopus and the Elsevier Bibliographic databases. The manuscript management system is completely online and includes a very quick and fair peer-review system, which is all easy to use. Visit http://www.dovepress.com/ testimonials.php to read real quotes from published authors. 